\title{
Sepsis causes reduction of insulin growth factor type 1 but not free triiodothyronine in patients receiving total parenteral nutrition
}

\author{
J. Gomez and K. Page \\ Nutrition Support Team, Sheffield Teaching Hospitals, Sheffield S10 2JF, UK
}

A potential role for insulin growth factor type 1 (IGF-1) and free triiodothyronine (FT3) as nutritional markers has been previously been suggested $^{(1,2)}$. Sepsis, however, is a common occurrence in hospitalised patients, and nutrition and sepsis have antagonist effects on these two hormones. Up to this date, it has not been studied how sepsis affects serum concentration of IGF-1 and FT3 in patients who are on total parenteral nutrition (TPN).

IGF-1 is a peptide hormone produced in cells throughout the body in response to circulating growth hormone concentration. It stimulates glucose and amino acids transport into cells, increases glucose oxidation in adipose tissues and inhibits lipolysis ${ }^{(3)}$. Its function is, thus, intimately related to growth and healing of tissues. But, serum IGF-1 concentration also responds to the intake of macronutrients: it is reduced during dietary restriction and increases again during refeeding.

Thyroid hormones play a significant metabolic role in energy homeostasis through the regulation of thermogenesis, lipid metabolism and fatty acids oxidation ${ }^{(4)}$. Tetraiodothyronine (T4) is the main hormone produced by the thyroid gland, but T3 is several times more potent. The type-1 5'-deiodinase enzyme (D1) facilitates the peripheral conversion of T4 to T3. Likewise IGF-1, calorie deprivation also suppresses FT3 production mainly mediated by inhibition of D1.

Hypermetabolic sepsis and critical illness reduce both serum IGF-1 and FT3. Endotoxins and cytokines have been implicated in the pathogenesis, but the metabolic routes leading to the inhibition of these two hormones appear to be different: inhibition of the Tyrosinekinase Janus-kinase (JAK) and Signal Transducer and Activator of Transcription (STAT) pathway leads to a suppression of IGF-1 mRNA, while central inhibition of the hypothalamic thyroid releasing hormone (TRH) and peripheral reduction of the activity of D1 are the main mechanisms for FT3 reduction ${ }^{(5,6)}$.

We, therefore, decided to study the effect of sepsis on IGF-1 and FT3 in patients requiring TPN.

Fifty-six patients on TPN were monitored (27 from ITU and 29 from other hospital wards). Of those, six surgical cases developed sepsis (two of them on ITU), and four of these had their TPN temporally stopped. IGF-1 and FT3 serum concentration was determined during sepsis and in the first sample that had been collected before its onset.

All six patients who had sepsis had marked lower IGF-1 in the second specimen, while four had lower and two higher FT3. We concluded that sepsis significantly reduced IGF-1 serum concentration $(t(5)=3.16, P<0.05)$ with a mean difference between samples of $27.6 \mu \mathrm{mol} / \mathrm{l}(5.2-50.1)$. The mean difference between samples was also lower for FT3 (0.03 pmol/l) but it was statistically non-significant. FT3 decreased in the second sample of both patients who continued TPN after the onset of sepsis, but it increased in three out of the four patients in whom TPN was stopped. Therefore, we could not establish any association between administration of TPN and FT3 serum concentration during sepsis.

In this first study on the effect of sepsis on IGF-1 and FT3 in TPN patients, we conclude that it has predictable effect on IGF-1 but not on FT3. These results should be taken into consideration for the interpretation of future research about the potential use of these hormones as nutritional markers, particularly if the participants develop sepsis during the collection of data.

1. Fontana L, Klein S et al. (2006) J Clin Endocrinol Metab 91, 3232-3235.

2. Donahue SP \& Phillips LS (1989) Am J Clin Nutr 50, 962-969.

3. Fang J, Mao D et al. (2006) Growth Horm IGF Res 16, 318-325.

4. Silva JE (2006) Physiol Rev 86, 435-464.

5. Fliers E, Guldenaar SE et al. (1997) J Clin Endocrinol Metab 82, 4032-4036.

6. Torpy DJ, Tsigos C et al. (1998) Metabolism 47, 1289-1293. 